УДК 911.2:591.9

\title{
BIODIVERSITY OF XYLOFAGOUS AND PREDATORY BEETLES (COLEOPTERA) OF THE VALLEY OF THE SOUTHERN BUG (UKRAINE)
}

\author{
W. B. Jędryczkowski \\ University of Ecology and Management, \\ Wawelska Str.? 14, 02-061 Warszawa, Poland
}

Results of preliminary research on beetle's biodiversity, which had been done in the vicinity of Winnica Region (Ukraine), are here presented. The following families represented by 55 species were recorded: Cerambycidae, Cantharidae, Cleridae, Coccinellidae, Elateridae and Malachiidae.

Key words: biodiversity, Southern Bug, Coleoptera, Cantharidae, Cerambycidae, Cleridae, Coccinellidae, Elateridae, Malachiidae.

Special thanks are due to Dr. Olena Cvietowa from the Institute of Hydraulic Engineering and Land Reclamation Ukrainian Academy of Agrarian Science, Kyiv. Without her help and assistance the field investigations could not be completed.

The program was sponsored by the University of Ecology and Management in Warsaw (grand No 0340).

During a field expedition, in year 2010, to the valley of Southern Bug in Ukraine, several samples of beetles (Coleoptera) have been collected. They are represented by six families of different ecological and biological character. On the base of this small collection, a preliminary report on beetles biodiversity is here presented. Since additional field expeditions are planed in near future, only some general remarks about collected families have been done.

This paper is based on the bigest project executing by the author and concerning biodiversity of river valleys in Poland and Ukraine.

Beetles were collected by entomological net. On each plots, about two hundred strokes have been executed. Insects were stored dry for laboratory study and are deposited partly in privet collection of the author and partly in the Museum and Institute of Zoology PAS in Warsaw.

The basic field research were located on six plots (tab. 1) in the Winnica region, the middle of Southern Bug Valley. More detail characteristics has been done by Dyguś and Jędryczkowski (in print).

About three hundred specimens of insects have been collected during field expedition. They were consisted, mostly by zoophagous and xylophagous species.

(C) Jędryczkowski W. B., 2013 
Biodiversity of xylofagous and predatory beetles...

Characteristics of the studied plots

\begin{tabular}{|l|c|c|c|c|}
\hline \multicolumn{1}{|c|}{ Locality } & Symbols & Latitude & Longitude & Biotopes \\
\hline Gnivan & Gn & 49,094 & 28,358 & 1,2 \\
\hline Komorovo & Ko & 49,144 & 28,375 & 3 \\
\hline Mogilovka & Mo & 49,091 & 28,313 & 2 \\
\hline Tyrov & Ty & 49,030 & 28,494 & 5 \\
\hline Urozhnoie & Ur & 49,149 & 28,334 & 5 \\
\hline Voroshylovka & Vo & 49,053 & 28,329 & 4 \\
\hline
\end{tabular}

Xylophagous species were represented by Cerambycidae family to which belong 13 species (tab. 2). They mostly species of wide range of distribution but some of them, like Agapanthia cardui and Theophilea subcylindricollis, develop in grasslands and meadows. It is necessary to underline, that mention above $T$. subcylindricollis was a dominate specie of this family.

Table 2

Species composition the Cerambycidae of the studied areas

\begin{tabular}{|l|c|c|c|}
\hline \multicolumn{1}{|c|}{ Species } & Count & Locality & Biotope \\
\hline Agapanthia cardui (L.) & 38 & $\begin{array}{c}\text { Gn, Ko, Ty, } \\
\text { Ur, Vo }\end{array}$ & $2,3,4,5$ \\
\hline Agapanthia villosoviridescens (De Geer) & 1 & Gn & 2 \\
\hline Alosterna tabacicolor (De Geer) & 6 & Gn, Mo & 2 \\
\hline Anastrangalia dubia (Scopoli) & 1 & Ur & 5 \\
\hline Anoplodera sexguttata (Fabricius) & 1 & Gn & 2 \\
\hline Dinoptera collaris (L.) & 6 & Gn, Mo & 2 \\
\hline Leiopus nebulosus (L.) & 1 & Gn & 1 \\
\hline Mesosa nebulosa (Fabricius) & 1 & Mo & 2 \\
\hline Paracorymbia maculicornis (De Geer) & 1 & Gn & 1 \\
\hline Phymatodes testaceus (L.) & 2 & Gn & 1 \\
\hline Phytoecia cylindrica (L.) & 1 & Gn & 2 \\
\hline Rhagium mordax (De Geer) & 1 & Gn & 2 \\
\hline Theophilea subcylindricollis Hladil & 15 & Ko, Ty, Ur, Vo & $3,4,5$ \\
\hline
\end{tabular}

Abbreviations: 1 - Ecotones, 2 - Broadleaf forest, 3 - Moisture meadows, 4 Riverside meadows, 5 - Xerothermic grass.

Predatory beetles were represented by five families. Cantharidae were represented by seven species (tab. 3) of wide geographical distribution and very frequent in many biotopes. Cantharis livida and Cantharis rufa were dominants in the family.

Family of Coccinellidae were represented by 15 species (tab. 4). They are feeding on small phytophagous insects, mainly aphids and young stadiums of Chryso- 
melidae. All collected species are distributed all over Europe and, in some case, in all Palaearctic (Coccinella septempunctata). Two dominant species, Psyllobora vigintiduopunctata and Tytthaspis sedecimpunctata, represent typical element of moisture biotope (Burakowski at all 1986) and were collected mainly on meadows.

Table 3

Species composition the Cantharidae of the studied areas

\begin{tabular}{|l|c|c|c|}
\hline \multicolumn{1}{|c|}{ Species } & Count & Locality & Biotope \\
\hline Cantharis fusca L. & 3 & Mo, Ur & 2,5 \\
\hline Cantharis lateralis L. & 5 & Vo & 4 \\
\hline Cantharis livida L. & 15 & Gn, Ko, Mo & $1,2,3,5$ \\
\hline Cantharis nigricans Müller & 7 & Gn, Mo, Ur & 1,5 \\
\hline Cantharis rufa L. & 11 & Ko, Vo & 3,4 \\
\hline Cantharis rustica Fallén & 7 & Ko, Ur, Vo & $3,4,5$ \\
\hline Rhagonycha lignosa (Müller) & 3 & Gn, Mo & 2 \\
\hline
\end{tabular}

Table 4

Species composition the Coccinellidae of the studied areas

\begin{tabular}{|l|c|c|c|}
\hline \multicolumn{1}{|c|}{ Species } & Count & Locality & Biotope \\
\hline Adalia bipunctata (L.) & 9 & Gn & 1,2 \\
\hline Adalia decempunctata (L.) & 1 & Gn & 1 \\
\hline Coccinella quinquepunctata L. & 1 & Gn & 2 \\
\hline Coccinella septempunctata L. & 3 & Gn & 1,2 \\
\hline Hippodamia variegata (Goeze) & 1 & Mo & 2 \\
\hline Hyperaspis reppensis (Herbst) & 1 & Ty & 5 \\
\hline Nephus bipunctatus (Kugelann) & 4 & Ty, Vo & 4,5 \\
\hline Oenopia conglobata (L.) & 1 & Gn & 1 \\
\hline Platynaspis luteorubra (Goeze) & 1 & Ur & 5 \\
\hline Propylea quatuordecimpunctata (L.) & 3 & Gn, Ko & 1,3 \\
\hline Psyllobora vigintiduopunctata (L.) & 14 & Gn, Mo, Ur, Vo & $1,2,4,5$ \\
\hline Scymnus frontalis (Fabricius) & 1 & Vo & 4 \\
\hline Scymnus interruptus (Goeze) & 1 & Gn & 1 \\
\hline Scymnus rubromaculatus (Goeze) & 4 & Gn, Mo, Vo & $1,2,4$ \\
\hline Tytthaspis sedecimpunctata (L.) & 10 & Ko, Ty, Vo & $3,4,5$ \\
\hline
\end{tabular}

Abbreviations: 1 - Ecotones, 2 - Broadleaf forest, 3 - Moisture meadows, 4 Riverside meadows, 5 - Xerothermic grass.

To family Elateridae belong species partly predatory and partly phytophagous. In general larval stadium of developing are mostly predatory or polyphagous animals. Since larvae of this family develop for several years in soil or decaying wood, they are very sensitive for even small changes in environment conditions. As such, this 
Biodiversity of xylofagous and predatory beetles...

ISSN 2078-6441. Вісник Львівського університету. Серія географічна. 2013. Випуск 46

family is considered a good indicator of environment changes $[12,13]$. This family was represented by 12 species (tab. 5) with Agriotes sputator and Cidnopus pilosus as dominant species, which are typical in meadows and grasslands.

Table 5

Species composition the Elateridae of the studied areas

\begin{tabular}{|l|c|c|c|}
\hline \multicolumn{1}{|c|}{ Species } & Count & Locality & Biotope \\
\hline Actenicerus siaelandicus (Müller) & 2 & $\mathrm{Vo}$ & 4 \\
\hline Agriotes lineatus (L.) & 1 & $\mathrm{Vo}$ & 4 \\
\hline Agriotes sputator (L.) & 14 & $\mathrm{Gn}, \mathrm{Ko}, \mathrm{Ur}, \mathrm{Vo}$ & $2,3,4,5$ \\
\hline Agrypnus murinus (L.) & 9 & $\mathrm{Gn}, \mathrm{Mo}, \mathrm{Ur}$ & $1,2,5$ \\
\hline Athous haemorrhoidalis (Fabricius) & 3 & $\mathrm{Gn}, \mathrm{Ko}, \mathrm{Mo}$ & 2,3 \\
\hline Athous jejunus Kiesenwetter & 1 & $\mathrm{Gn}$ & 2 \\
\hline Athous vittatus (Gmelin) & 4 & $\mathrm{Mo}$ & 2 \\
\hline Cidnopus pilosus (Leske) & 13 & $\mathrm{Ur}$ & 5 \\
\hline Hemicrepidius niger (L.) & 1 & $\mathrm{Gn}$ & 1 \\
\hline Pheletes aeneoniger (De Geer) & 3 & $\mathrm{Mo}$ & 2 \\
\hline Prosternon tessellatum (L.) & 1 & $\mathrm{Ur}$ & 5 \\
\hline Synaptus filiformis (Fabricius) & 3 & $\mathrm{Gn}$ & 1 \\
\hline
\end{tabular}

Abbreviations: 1 - Ecotones, 2 - Broadleaf forest, 3 - Moisture meadows, 4 Riverside meadows, 5 - Xerothermic grass.

Family Cleridae was represented there only by three species (tab. 6) which are common all over Europe. Their larvae pray on wood drilling insects or parasite bees nests but adults feed on pollen and occasionally on small insects [4].

Table 6

Species composition the Cleroidea of the studied areas.

\begin{tabular}{|c|c|c|c|}
\hline Species & Count & Locality & Biotope \\
\hline \multicolumn{4}{|c|}{ Cleridae } \\
\hline Korynetes caeruleus (De Geer) & 4 & $\mathrm{Gn}$ & 1 \\
\hline Tillus elongatus (L.) & 2 & $\mathrm{Gn}$ & 1 \\
\hline Trichodes apiarius (L.) & 2 & Gn & 1 \\
\hline \multicolumn{4}{|c|}{ Malachiidae } \\
\hline Anthocomus equestris (Fabricius) & 4 & Gn & 1 \\
\hline Axinotarsus pulicarius (Fabricius) & 2 & Gn & 2 \\
\hline Clanoptilus geniculatus (Germar) & 21 & Gn, Ko, Ty, Vo & $1,3,4,5$ \\
\hline Malachius aeneus (L.) & 1 & Ko & 3 \\
\hline Malachius bipustulatus (L.) & 20 & $\mathrm{Gn}, \mathrm{Ko}, \mathrm{Ur}$ & $2,3,5$ \\
\hline
\end{tabular}

Abbreviations: 1 - Ecotones, 2 - Broadleaf forest, 3 - Moisture meadows, 4 Riverside meadows, 5 - Xerothermic grass. 
Family Malachiidae is represented by five species (tab. 6). The interesting one is Clanoptilus geniculatus, a rare species occurring in open landscapes such as steppes and meadows. Larvae of Malachiidae are predacious feeding mainly on xylophages. Adults specimens, usually feeds on pollen, fungi and small insects which visit flowers [10].

The material presented here, is far to be representative for this region. Some species such as Theophilea subcylindricollis (Cerambycidae), Hippodamia variegata (Coccinellidae) or Actenicerus siaelandicus and Synaptus filiformis (Elateridae) indicate, that studied area preserve moisture character typical for natural fresh meadows.

1. Burakowski B., Mroczkowski M., Stefańska H. 1986. Cucujoidea, część 2. Katalog fauny Polski, Warszawa, część XXIII, Chrząszcze Coleoptera, 13, 278 pp.

2. Dyguś K. H., Senchyna B., Jędryczkowski W. B. 2011. Anthropogenic Changes of Vegetation Cover and Entomofauna in the Dnieper Terrace near Kyiv. In [Ed. K.H. Dyguś] The Water Environment. Problems of Evaluation and Protection. Monografie WSEiZ. Warszawa, 289-297.

3. Dyguś K. and Jędryczkowski W. 2013. Bioróżnorodność doliny Południowego Bugu na przykładzie Wybranych powierzchni podola w obwodzie winnickim (Ukraina).

4. Gerstmeier R. 1998. Checkered Beetles. Illustrated Key to the Cleridae of the Western Palaearctic. Margraf Verlag, Weikersheim, $241 \mathrm{pp}, 8$ plates.

5. Jędryczkowski W. B., Kupryjanowicz J. 2005. Biegaczowate, Carabidae (chrząszcze, Coleoptera) czterech Środowisk Biebrzańskiego Parku Narodowego. [Beetles (Coleoptera: Carabidae) of four biotopes of the Biebrza National Park]. [In:] Przyroda Biebrzańskiego Parku Narodowego. Osowiec-Twierdza, 325-329.

6. Jędryczkowski W. B., Jarząbek-Żukowska A. 2007. Różnorodność gatunkowa chrząszczy epigeicznych (Coleoptera) terenów zalewowych Dolnego Bugu. [Biodiversity of the epigeic beetles (Coleoptera) of the flooded terrain of Lower Bug River] VIII Międzynarodowa Konferencja Naukowa "Zagospodarowanie zlewni Bugi i Narwi w ramach zrównoważonego rozwoju". WSEiZ.

7. Jędryczkowski W. B. 2010. Biodiversity of the Predatory Beetles (Coleoptera) in the Valley of Bug River in Poland. In [Ed. K.H. Dyguś'] Natural Environment of Transfrontier River Catchments in Poland and Ukraine. Monografie WSEiZ. Warszawa, 97-104.

8. Jędryczkowski W. B. 2010b. Shield-Bugs (Hemiptera: Pentatomoidea) of the Central Bug River Valley. (Preliminary report). In [Ed. K.H. Dyguś] Natural Environment of Transfrontier River Catchments in Poland and Ukraine. Monografie WSEiZ. Warszawa, 105108.

9. Jędryczkowski W. B. 2012. Delata Donu. Wiadomości Uczelniane WSEiZ, Warszawa, 1: 32-33.

10. Kolibáč J., Majer K., Švihla V. 2005. Cleroidea. Beetles of the superfamily Cleroidea in the Czech and Slovak Republics and neighboring areas. Clarion Production, Praha, $186 \mathrm{pp}$.

11. Kubisz D., Jędryczkowski W. B., Ścibior R. 2010. Species Diversity of Leaf-Beetles (Coleoptera: Chrysomelidae) of the Bug River Valley. In [Ed. K.H. Dyguś] Natural Environment of Transfrontier River Catchments in Poland and Ukraine. Monografie WSEiZ. Warszawa, 81-96.

12. Laibner S. 2000. Elateridae of the Czech and Slovak Republics. Zlin, 292 pp. 
Biodiversity of xylofagous and predatory beetles...

13. Tarnawski D. 2000. Elateridae - Sprężykowate (Insecta: Coleoptera) Część I. Fauna Polski, Warszawa, 21: $412 \mathrm{pp}$.

Стаття: надійшла до редколегії 16.05.2013 доопрацьована 12.07.2013

прийнята до друку 25.09.2013

\section{БIOPIЗНОМАНІТТЯ XYLOFAGOUS I ХИЖИХ ЖУКІВ (COLEOPTERA) ДОЛИНИ ПІВДЕННОГО БУГУ (УКРӒ̈НА)}

\section{В. Б. Едричковскі}

Університет екологї та управління, вул. Вавельська, 14, 02-061 Варшава, Польща

Представлено результати попереднього вивчення біорізноманіття жуків Вінницької області. Визначено 55 видів жуків таких родин: Cerambycidae, Cantharidae, Cleridae, Coccinellidae, Elateridae i Malachiidae.

Ключові слова: біорізноманіття, Південний Буг, Coleoptera, Cantharidae, Cerambycidae, Cleridae, Coccinellidae, Elateridae, Malachiidae.

\section{БИОРАЗНООБРАЗИЕ ХYLОFАGОUS И ХИЩНЫЙ ЖУКОВ (COLEOPTЕRА) ДОЛИНЫ ЮЖНОГО БУГА (УКРАИНА)}

\section{В. Б. Едрычковски}

Университет экологии и управления, ул. Вавельская, 14, 02-061 Варшава, Польша

Представлено результаты предварительного изучения биоразнообразия жуков Винницкой области. Определено 55 видов жуков таких семейств: Cerambycidae, Cantharidae, Cleridae, Coccinellidae, Elateridae и Malachiidae.

Ключевые слова: биоразнообразие, Южный Буг, Coleoptera, Cantharidae, Cerambycidae, Cleridae, Coccinellidae, Elateridae, Malachiidae. 